University of Wollongong

Research Online

Faculty of Informatics - Papers (Archive)

Faculty of Engineering and Information

Sciences

$1-1-2011$

\title{
Modelling carbon nanostructures for filtering and adsorbing polycyclic aromatic hydrocarbons
}

Thien Tran-Duc

University of Wollongong, thien@uow.edu.au

Ngamta Thamwattana

University of Wollongong, ngamta@uow.edu.au

Follow this and additional works at: https://ro.uow.edu.au/infopapers

Part of the Physical Sciences and Mathematics Commons

\section{Recommended Citation}

Tran-Duc, Thien and Thamwattana, Ngamta: Modelling carbon nanostructures for filtering and adsorbing polycyclic aromatic hydrocarbons 2011, 2072-2077.

https://ro.uow.edu.au/infopapers/1275

Research Online is the open access institutional repository for the University of Wollongong. For further information contact the UOW Library: research-pubs@uow.edu.au 


\title{
Modelling carbon nanostructures for filtering and adsorbing polycyclic aromatic hydrocarbons
}

\begin{abstract}
The discovery of carbon nanostructures, such as graphene and carbon nanotubes, has led to the creation of many novel nano-devices. In this paper, we consider an environmental application of carbon nanostructures for filtering and adsorbing polycyclic aromatic hydrocarbons (PAHs) which are environmental pollutants. We mathematically investigate the adsorption of PAHs onto a graphene sheet and determine the underlying mechanisms of suction of PAHs into a carbon nanotube. We adopt a continuum approach together with the semi-empirical Lennard-Jones potential to determine van der Waals interaction forces and energies. Results obtained here for certain PAHs are well in agreement with existing experimental and theoretical results. We comment that the use of elementary mechanical principles and classical applied mathematical modelling techniques in this paper enables the formulation of explicit analytical criteria and ideal model behaviour for problems in nanotechnology for which previously only experiments and molecular dynamics simulations were available. Further the method presented here is less computationally intensive compared with conventional methods, such as ab initio calculations and density functional theory, making it an ideal approach for modelling molecules with large numbers of atoms, such as PAHs, carbon nanostructures and biomolecules.
\end{abstract}

\section{Keywords}

polycyclic, adsorbing, aromatic, filtering, modelling, nanostructures, carbon, hydrocarbons

\section{Disciplines}

Physical Sciences and Mathematics

\section{Publication Details}

Tran-Duc, T. \& Thamwattana, N. (2011). Modelling carbon nanostructures for filtering and adsorbing polycyclic aromatic hydrocarbons. Journal of Computational and Theoretical Nanoscience, 8 (10), 2072-2077. Original item available here 
Journal of Computational and Theoretical Nanoscience
vol 8, pp. 2072-2077, (2011).

Modelling carbon nanostructures for filtering and absorbing polycyclic aromatic hydrocarbons

\author{
Thien Tran-Duc and Ngamta Thamwattana \\ Nanomechanics Group, School of Mathematics and Applied Statistics, \\ University of Wollongong, Wollongong, NSW 2522, Australia.
}

Email: ttd689@uow.edu.au. Tel: +6124221 8192. Fax: +61242214845

January 12, 2011

\begin{abstract}
The discovery of carbon nanostructures, such as graphene and carbon nanotubes, has led to the creation of many novel nano-devices. In this paper, we consider an environmental application of carbon nanostructures for filtering and absorbing polycyclic aromatic hydrocarbons (PAHs) which are environmental pollutants. We mathematically investigate the absorption of PAHs onto a graphene sheet and determine the underlying mechanisms of suction of PAHs into a carbon nanotube. We adopt a continuum approach together with the semiempirical Lennard-Jones potential to determine van der Waals interaction forces and energies. Results obtained here for certain PAHs are well in agreement with existing experimental and theoretical results. We comment that the use of elementary mechanical principles and classical applied mathematical modelling techniques in this paper enables the formulation of explicit analytical criteria and ideal model behaviour for problems in nanotechnology for which previously only experiments and molecular dynamics simulations were available. Further the method presented here is less computationally intensive compared with conventional methods, such as $a b$ initio calculations and density functional theory, making it an ideal approach for modelling molecules with large numbers of atoms, such as PAHs, carbon nanostructures and biomolecules.
\end{abstract}

Keywords graphene, carbon nanotubes, polycyclic aromatic hydrocarbons, van der Waals interaction, Lennard-Jones potential

\title{
1 Introduction
}

Polycyclic aromatic hydrocarbons (PAHs) are formed as byproducts in incomplete combustion. In volcanic eruptions and forest fires, PAHs are released heavily into the environment. PAHs are also created in human activities, such as fuels burning in vehicles, aircrafts and factories. PAHs are 
found ubiquitously in air, water, soil and even in foods, especially in broiled foods. They can get into the human body via breathing the air, consuming food, drinking water, or through skin contact. Once inside the human body, PAHs normally remain in tissues containing fat, in the kidneys or the liver. Degradation of PAHs in the body can create new substances causing cancer, mutagen and teratology [1]. Therefore, they are categorized as environmental pollutants [2]. Another environmental pollutant is soot. Similar to PAHs, soot particles are also formed in incomplete burnings. Sizes of soot particles range widely from twenty nanometers to more than several hundred nanometers in diameter $[3,4]$. They are ubiquitous in the air and therefore easily inhaled by humans. Soot particles can cause health hazards, such as lung diseases and in particular lung cancer [5]. In a study of the World Trade Center disaster on September 11th, 2001, Pleil et al. [6] estimate that more than one million tons of dust and smoke, containing PAHs and soot particles, was released into the air. Observations conducted shortly after the disaster show that birth defect rates in the offspring were much higher than normal and conclusively associated with the high level of PAHs and soot in the air. With this in mind, this paper aims to contribute to the development of devices which can be used to eliminate these pollutants from the environment.

In normal environmental conditions PAHs prefer to stay on the surface of soot particles [7] creating a further environmental issue. Structurally, soot particles comprise layered carbon atoms whose structures are similar to graphene sheets [8]. PAHs comprise fused rings which are structurally similar to benzene rings and they are normally thought of as fragments of a graphene sheet (see Figure 1 for examples of PAHs). Van der Waals interactions between atoms are recognized as the driving force for adsorption of PAHs onto soot particles $[9,10]$. A model which is popularly used in most studies is the interaction between PAHs and a graphene sheet $[8,9,10]$. Conventional methods, such as $a b$ initio calculations and density functional theory, becomes too computationally expensive and seem infeasible in this setting [8] due to the large size of the molecules involved. Therefore, most studies only deal with small PAHs and an approximation of a graphene sheet. Kubicki [9] employs ab initio calculations to model the van der Waals interaction between some small PAHs (benzene, naphthalene, fluorene, anthracene, phenanthrene, pyrene and fluoranthene) and the approximation of $\mathrm{C}_{24} \mathrm{H}_{12}$ (coronene) for the graphene sheet. Although the results obtained are consistent with their experimental results on the same models, $\mathrm{C}_{24} \mathrm{H}_{12}$ seems rather small to be used as an approximation for a graphene sheet [8] and therefore the results may not reflect the true values of the interaction between PAHs and a graphene sheet. Collignon et al. [8] also state that a full $a b$ initio calculation is not feasible due to the large size of the molecules and therefore these authors adopt a semi-empirical model which allows calculations for larger approximations of a graphene sheet. In [8], the authors 


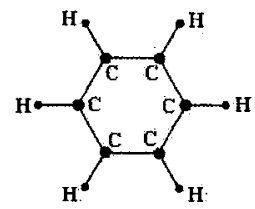

(a) Benzene

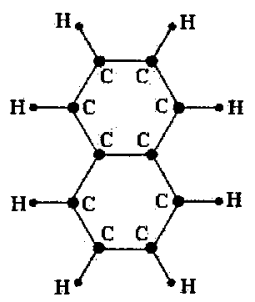

(b) Naphthalene

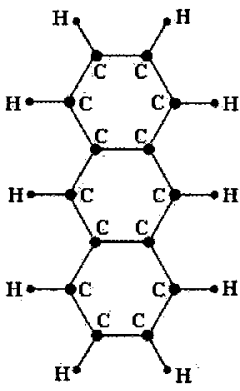

(c) Anthracene

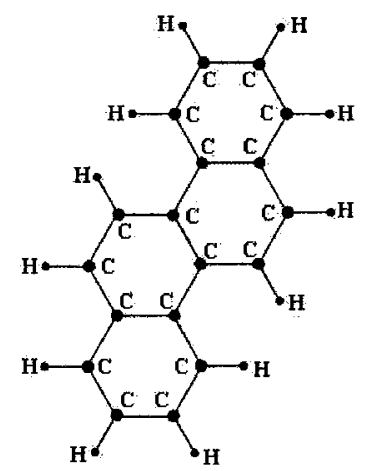

(d) Chrysene

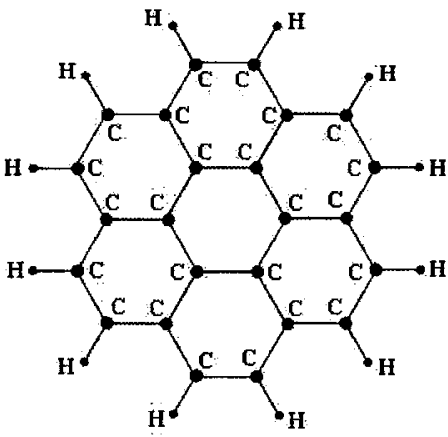

(e) Coronene

Figure 1: Some PAHs.

combine "a self-consistent field interaction energy calculation at the semiempirical AM1, PM3, or MNDO level of approximation for the electrostatic contribution" and "an empirical dispersion term" to calculate interaction energies between benzene and some approximations of a graphene sheet including $\mathrm{C}_{24} \mathrm{H}_{12}, \mathrm{C}_{80} \mathrm{H}_{22}$ and $\mathrm{C}_{150} \mathrm{H}_{30}$. Results in [8] point out that $\mathrm{C}_{24} \mathrm{H}_{12}$ is too small to approximate a graphene sheet, while results for $\mathrm{C}_{150} \mathrm{H}_{30}$ are more consistent with those shown in $[11,12]$. In another work, ChakarovaKäck et al. [10] improve the standard density functional theory (DFT) for more general geometries in order to perform calculations for large size molecules. Chakarova-Käck et al. [10] correct "the correlation part of the energy of a standard self-consistent DFT calculation" and then add a nonlocal correlation energy to obtain the final interaction energy. Results for benzene adsorbed on an approximation $(5 \times 5)$ unit cells of the graphene sheet and naphthalene on an approximation $(6 \times 5)$ unit cells of a graphene sheet agree well with experimental results of $[13,14]$. However, these methods are still too computationally intensive and they are almost unfeasible to perform calculations for a bigger system which involves large sized molecules, 


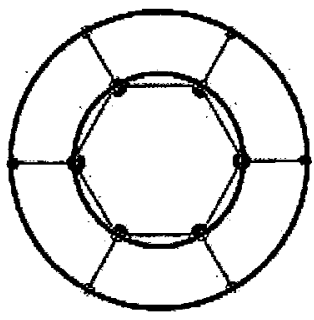

(a) Benzene

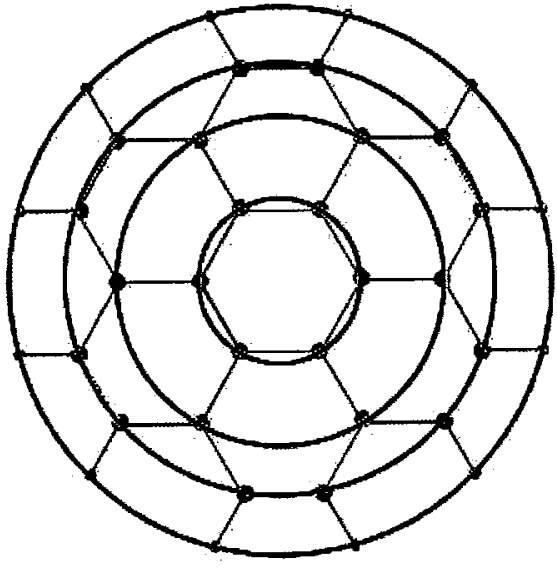

(b) Coronene

Figure 2: Continuous models for benzene and coronene.

such as PAH clusters, carbon nanostructures and biomolecules. Therefore, a less expensive computational method is necessary. For this purpose, the semi-empirical Lennard-Jones potential function combined with a continuous model appears to be a good candidate [15], and this is the method adopted in this paper. Mathematical details for this approach are given Sections 2 and 3.

The phenomenon that PAHs are adsorbed onto soot particles in normal conditions in the environment suggests using carbon nanostructures as environmental materials to filter PAHs. In this paper, using a continuum approach and the Lennard-Jones potential we investigate the mechanisms of PAHs being absorbed onto a graphene sheet and being attracted into a carbon nanotube. In this approach a graphene sheet and a carbon nanotube are modelled as an infinite planar surface and a semi-infinite cylindrical tube, respectively. For PAHs, as an example, this paper only considers benzene and coronene. We note that these PAHs are symmetric and thus we use concentric circular rings to model the fused rings, as shown in Figure 2. We note that the outermost rings of benzene and coronene comprise hydrogen atoms, while the inner rings are made up of carbon atoms.

In the following section we present mathematical model and computational results for the suction of PAHs into carbon nanotubes. Section 3 details mathematical model and provides results for the absorption of PAHs onto a graphene sheet. Finally, conclusions are given in Section 4. 


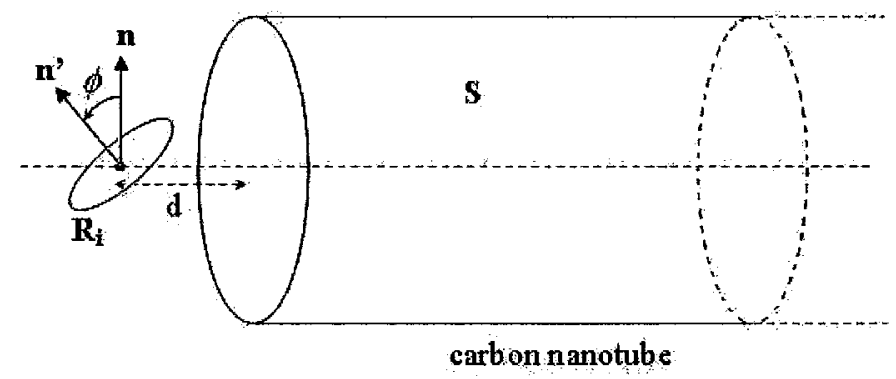

Figure 3: Interaction between a ring and a carbon nanotube.

\section{Carbon nanotubes for suction of PAHs}

For simplicity, here we only consider symmetric PAHs, such as benzene and coronene. Each PAH can be modelled as a combination of circular rings (Figure 2) and the total interaction energy of a PAH and a carbon nanotube can be obtained as a sum of the interaction energy of each ring with the nanotube. If we assume that the centre of the PAHs is on the axis of the carbon nanotube, the problem reduces to only finding the interaction energy between an on-axis circular ring and a tube. The schematic for our model is illustrated in Figure 3. We use an rotational angle $\phi$ and a horizontal distance $d$ from the centre of the ring to the open end of the tube to describe relative positions between the ring and the tube.

The total interaction energy between a PAH and a carbon nanotube is given by

$$
E_{c}=\sum_{i=0}^{k} E_{i},
$$

where $E_{i}$ is the interaction energy of each ring with the carbon nanotube and $k$ is the number of rings in a $\mathrm{PAH}$, for example $k=2$ for a benzene molecule and $k=4$ for a coronene molecule. $E_{i}$ can be evaluated as follows

$$
E_{i}=\eta_{i} \eta_{T} \int_{R_{i}} \int_{S}\left(-\frac{A}{\rho^{6}}+\frac{B}{\rho^{12}}\right) d S d \ell,
$$

where $\eta_{i}$ and $\eta_{T}$ are the atomic line density of ring $R_{i}$ and the surface density of the carbon nanotube, respectively. Throughout this paper, we define the atomic line density as the number of atoms on the ring divided by the circumference of the ring and similarly, the atomic surface density can be found from dividing the number of atoms on the surface by the surface area. $A$ and $B$ represent the attractive and repulsive constants, which can be determined from $A=4 \epsilon \sigma^{6}$ and $B=4 \epsilon \sigma^{12}$, where $\epsilon$ and $\sigma$ are the energy well depth and the van der Waals diameter, respectively. We note that 
numerical values for constants used in our model are given in Table 1 . We also note that we use Lorentz-Berthelot mixing rules, $\epsilon_{C H}=\left(\epsilon_{C} \epsilon_{H}\right)^{1 / 2}$ and $\sigma_{C H}=\left(\sigma_{C}+\sigma_{H}\right) / 2$ for interactions between carbon and hydrogen atoms .

Table 1: Numerical values of constants used in the model.

\begin{tabular}{lcc}
\hline \hline Atomic surface density of a graphene sheet & 0.382 & $\AA^{-2}$ \\
Atomic surface density of a carbon nanotube & 0.382 & $\AA^{-2}$ \\
Radius of CNT $(4,9)$ & 4.514 & $\AA$ \\
Radius of CNT $(7,7)$ & 4.746 & $\AA$ \\
Radius of CNT $(8,8)$ & 5.424 & $\AA$ \\
Radius of CNT $(9,9)$ & 6.102 & $\AA$ \\
Radius of CNT $(10,10)$ & 6.780 & $\AA$ \\
Radius of CNT $(8,12)$ & 6.825 & $\AA$ \\
Radius of CNT $(10,11)$ & 7.122 & $\AA$ \\
Radius of CNT $(12,12)$ & 8.136 & $\AA$ \\
Energy well depth $[16]$ & & \\
$\quad \epsilon_{C}$ & 0.07 & $(\mathrm{kcal} / \mathrm{mol})$ \\
$\epsilon_{H}$ & 0.03 & $(\mathrm{kcal} / \mathrm{mol})$ \\
van der Waals diameter $[16]$ & & \\
$\quad \sigma_{C}$ & 3.55 & $\AA$ \\
$\sigma_{H}$ & 2.42 & $\AA$ \\
\hline \hline
\end{tabular}

In order to determine whether a molecule can be accepted by a carbon nanotube, we first consider the distribution of the van der Waals force along the axial direction which is given by

$$
F=-\frac{\partial E}{\partial Z}
$$

Figure 4 shows the axial van der Waals force for a benzene molecule interacting with a nanotube. We can see that near the open end of small carbon nanotubes there exist repulsive regions which prevent the molecule from entering the carbon nanotubes. These regions are symmetric about the open end of the carbon nanotubes. The two points at which the axial interaction force changes from positive (attractive) to negative (repulsive) and vice versa are denoted by $-Z_{0}<0$ and $Z_{0}>0$. The smaller the carbon nanotube the larger the repulsive region and therefore it becomes more difficult for the molecule to get into the carbon nanotube. The repulsive region becomes smaller for a bigger carbon nanotube and it starts to disappear as the carbon nanotube is sufficiently large. According to Cox et al. [17], the total work of the axial force from $-\infty$ to $Z_{0}$ is referred to as the acceptance energy, which is denoted by $W_{a}$,

$$
W_{a}=\int_{-\infty}^{Z_{0}} F d Z
$$




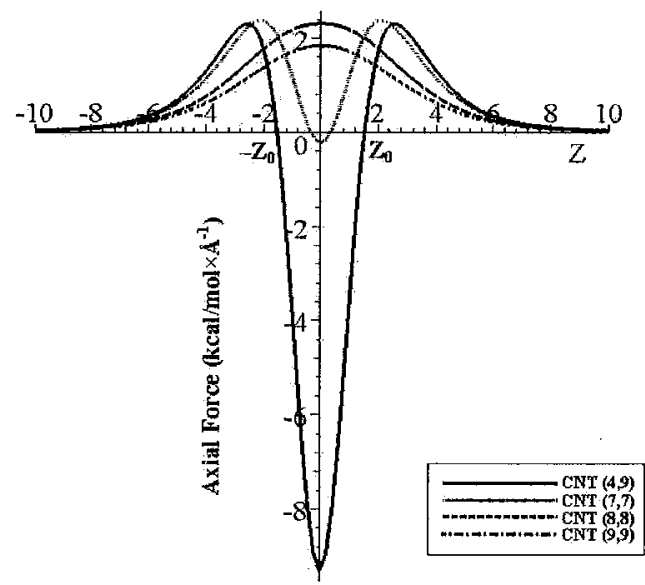

(a)

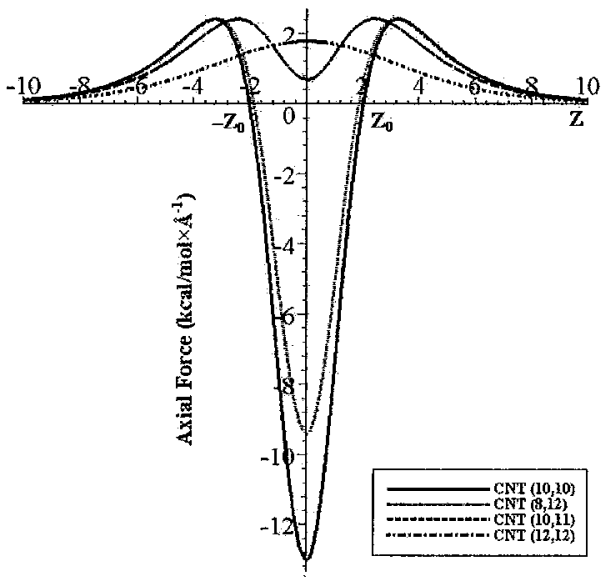

(b)

Figure 4: (a) Axial interaction force between benzene and carbon nanotubes $(4,9),(7,7),(8,8)$ and $(9,9)$ when $\phi=0$; (b) Axial interaction force between coronene and carbon nanotubes $(10,10),(8,12),(10,11)$ and $(12,12)$ when $\phi=0$.

Due to $\left.E\right|_{Z=\infty}=0$, equation (4) becomes

$$
W_{a}=-\left.E\right|_{Z=Z_{0}} .
$$

From equation (5), we can see that the acceptance energy is equal to the interaction energy at $Z_{0}$ but in the opposite sign. If we assume that the molecule has zero initial kinetic energy, then the molecule is only accepted into the carbon nanotube if and only if the acceptance energy is positive. For sufficiently large carbon nanotubes there is no repulsive region because the axial force is always positive and therefore the molecule is always accepted by the carbon nanotube. We refer to $r_{0}$ as the critical radius of the carbon nanotube which a molecule starts to become accepted. Table 2 shows computational results for $r_{0}$ for different orientations of benzene and coronene molecules. We see that when the rotational angle of these molecules increases, $r_{0}$ also increases. This implies that as the rotational angle increases, a larger sized nanotube is required to encapsulate these molecules. For example, from Table 2 we can see that for values of $\phi$ less than $\pi / 3$ benzene is accepted by a $(7,7)$ carbon nanotube, which has the radius of $4.746 \AA$, and when the tube radius is greater than $4.87 \AA$ benzene is always accepted into the tube regardless of its orientation. Similarly, a coronene molecule will not be accepted by a carbon nanotube with radius smaller than $6.91 \AA$, but will be accepted by a carbon nanotube which $r>7.20 \AA$, regardless of its orientation. However, we note that if the carbon nanotube is too large 
Table 2: Critical radii of carbon nanotubes for which molecules at certain orientations start to become accepted into the nanotubes.

\begin{tabular}{ccc}
\hline & $\begin{array}{c}\text { Rotational angle } \\
\phi\end{array}$ & $\begin{array}{c}\text { Critical radius } \\
r_{0}(\AA)\end{array}$ \\
\hline Benzene & & \\
& $90^{\circ}$ & 4.87 \\
& $60^{\circ}$ & 4.73 \\
& $45^{\circ}$ & 4.65 \\
Coronene & $30^{\circ}$ & 4.61 \\
& $0^{\circ}$ & 4.55 \\
& & \\
& $90^{\circ}$ & 7.20 \\
& $60^{\circ}$ & 6.98 \\
& $45^{\circ}$ & 6.93 \\
& $30^{\circ}$ & 6.92 \\
& $0^{\circ}$ & 6.91 \\
\hline
\end{tabular}

then the interaction force becomes too small to attract the molecule into the tube. Thus, an appropriate size of a carbon nanotube to be used in filtering applications is when its radius is equal to the size that a molecule starts being accepted, regardless of its orientation.

\section{Adsorption of PAHs onto a graphene sheet}

Similar to Section 2, the total interaction energy is the sum of interactions of all rings of $\mathrm{PAH}$ and the graphene sheet,

$$
E_{g}=\sum_{i=0}^{k} E_{i},
$$

where here $E_{i}$ is the interaction energy between ring $R_{i}$ and the graphene sheet and $E_{i}$ can be evaluated by taking a line and a surface integrals of the Lennard-Jones potential function over the ring $R_{i}$ and the graphene surface, namely

$$
E_{i}=\eta_{i} \eta_{G} \int_{R_{i}} \int_{S}\left(-\frac{A}{\rho^{6}}+\frac{B}{\rho^{12}}\right) d S d \ell
$$

where $\rho$ is the distance between a line element $d \ell$ of the ring $R_{i}$ and a surface element $d S$ of the graphene surface and it is specified by two parameters, namely a rotational angle $\phi$ and a vertical distance $d$ from the graphene sheet to the centre of the ring (Figure 5).

Our computational results show that a $\mathrm{PAH}$ is adsorbed onto a graphene sheet in three stages before reaching the most stable configuration. The first 


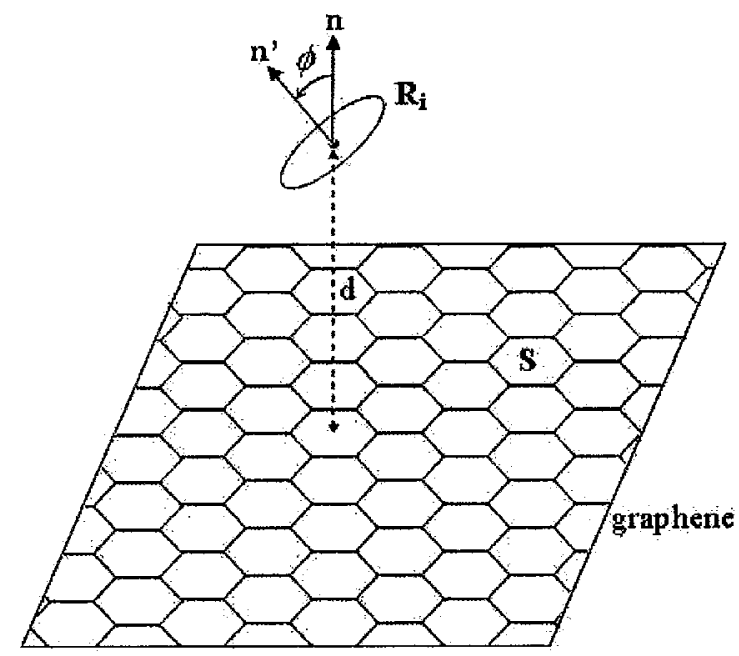

Figure 5: Interaction between a ring and a graphene sheet.

stage corresponds to a large distance away from the graphene sheet. In this stage, the perpendicular configuration, i.e the plane of the $\mathrm{PAH}$ forms a right angle with the graphene sheet, is more favorable because it minimizes the interaction energy. Tilted configuration appears in the second stage where the PAH is at an intermediate distance from the graphene sheet and finally we have the parallel configuration when the PAH is closed to the surface of graphene. The three stages occur in a decreasing order of the interaction energy as the PAH moves closer to the graphene sheet. We find that the most stable configuration is a parallel configuration, where the interaction energy is minimum.

For example, the first stage for the adsorption of coronene onto a graphene sheet corresponds to $d>7.5 \AA$, the second stage is for $d$ in the range 3.9 $7.5 \AA$, and the third stage is when $d \leqslant 3.9 \AA$. The corresponding energy decreases from zero to $-7.054 \mathrm{kcal} / \mathrm{mol}$ in the first stage, further reduces to $-26.122 \mathrm{kcal} / \mathrm{mol}$ in the second stage, and continues decreasing to -31.451 $\mathrm{kcal} / \mathrm{mol}$ at $d=3.51 \AA$ in the third stage before it starts to increase as the coronene gets too close to the graphene sheet. Since the parallel configuration at the distance of $3.51 \AA$ produces the lowest interaction energy, this is the equilibrium configuration for which coronene is most stable on a graphene sheet.

The finding that the parallel configuration is the most stable configuration in a PAH-graphene system is consistent with results of other authors. In a comprehensive study of $\pi-\pi$ systems (a PAH-graphene system is also called a $\pi-\pi$ system), Hunter [18] concludes that the parallel structure is the configuration which minimizes the van der Waals interaction energy. 
On using a semi-empirical method, Collignon et al. [8] find that benzene is stable on a graphene sheet with a parallel configuration at a distance in the range from $3.2 \AA$ to $3.4 \AA$ and the corresponding energy from $-9.8 \mathrm{kcal} / \mathrm{mol}$ to $-10.8 \mathrm{kcal} / \mathrm{mol}$. In another work, Chakarova-Käck et al. [10] employ the density functional theory to investigate an adsorption of benzene and naphthalene on graphite. These authors conclude that benzene and naphthalene are stable at the same distance of $3.6 \AA$ away from the graphite surface, but at different energy levels which are $-11.41 \mathrm{kcal} / \mathrm{mol}$ for benzene and -17.58 $\mathrm{kcal} / \mathrm{mol}$ for naphthalene. Zacharia et al. [19] conduct experiments in order to determine cohesive energy of PAHs on graphite using thermal desorption spectroscopy and results for the interaction energy for benzene, naphthalene and coronene are $-11.55 \pm 1.84,-18.48 \pm 2.30$ and $29.95 \pm 4.61 \mathrm{kcal} / \mathrm{mol}$, respectively. Table 3 shows that there is a good agreement between our computational results and those presented in other studies.

Table 3: Potential energies for certain PAHs interacting with a graphene sheet.

\begin{tabular}{lcc}
\hline \hline \multicolumn{1}{c}{ PAHs } & $\begin{array}{c}\text { Equilibrium distance } \\
(\AA)\end{array}$ & $\begin{array}{c}\text { Energy } \\
(\mathrm{kcal} / \mathrm{mol})\end{array}$ \\
\hline \hline Benzene $\left(\mathrm{C}_{6} \mathrm{H}_{6}\right)$ & 3.47 & -10.232 \\
Naphthalene $\left(\mathrm{C}_{10} \mathrm{H}_{8}\right)$ & 3.49 & -16.163 \\
Anthracene $\left(\mathrm{C}_{14} \mathrm{H}_{10}\right)$ & 3.49 & -22.097 \\
Chrysene $\left(\mathrm{C}_{18} \mathrm{H}_{12}\right)$ & 3.50 & -28.034 \\
Coronene $\left(\mathrm{C}_{24} \mathrm{H}_{12}\right)$ & 3.51 & -35.628 \\
\hline \hline
\end{tabular}

\section{Conclusions}

From the phenomenon that PAHs are generally adsorbed onto soot particles in normal environmental conditions, the idea of using carbon nanostructures, such as graphene sheets and carbon nanotubes, as pollutant cleaned-up materials is explored. In this paper, we employ a continuum model together with the semi-empirical Lennard-Jones potential to investigate the mechanics of PAHs adsorbed onto graphene sheets as well as attracted into carbon nanotubes. Computational results show that PAHs are generally adsorbed onto a graphene sheet in three stages, namely perpendicular, tilted and parallel configurations, depending on the distance between the PAHs and the graphene sheet. The parallel configuration is shown to be the equilibrium configuration as the interaction energy is minimum. The bigger the PAHs the larger the binding energy and therefore the more stable the PAH-graphene system. Our computational results also show that carbon nanotubes can accept horizontal PAHs $(\phi=0)$ more easily than PAHs with 
other orientations $(0<\phi \leqslant \pi / 2)$. Further, carbon nanotubes which accept PAHs with the perpendicular orientation will accept the PAHs with any orientation. The continuum approach adopted here is relatively simple and less computationally intensive compared with conventional methods, such as $a b$ initio calculations or density functional theory. With results that are in good agreement with these other studies, our approach presents a more efficient way of dealing with systems of large molecules, such as PAHs clusters, bimolecules and carbon nanostructures.

\section{References}

[1] F. P. Perera, Environment and cancer: Who are susceptible?, Science 278 (1997) 1068.

[2] B. J. Finlayson-Pitts, J. N. Pitts, Tropospheric air pollution: Ozone, airborne toxics, polycyclic aromatic hydrocarbons, and particles, Science 276 (1997) 1045.

[3] M. Alfe, B. Apicella, R. Barbella, A. Tregrossi, A. Ciajolo, Distribution of soot molecular wieght/size along premixed flames as inferred by size exclusion chromatography, Energy \& Fuels 21 (2007) 136-140.

[4] T. Kamimoto, M. Shimono, M. Kase, Dynamic measurements of soot aggregate size in diesel exhaust by a light scattering method, J. Phys.: Conf. Series 85 (2007) 012008.

[5] C. A. Pope, R. T. Burnett, M. J. Thun, E. E. Calle, D. Krewski, K. Ito, G. D. Thurston, Lung cancer, cardiopulmonary mortality and long-term exposure to fine particle air pollution, JAMA 287(9) (2002) 1132-1141.

[6] J. D. Pleil, A. F. Vette, B. A. Johnson, S. M. Rappaport, Air levels of carcinogenic polycyclic aromatic hydrocarbons after the world trade center disaster, PNAS 101(32) (2004) 11685-11688.

[7] S. L. Manzello, D. B. Lenhert, A. Yozgatligil, M. T. Donovan, G. W. Mulholland, M. R. Zachariah, W. Tsang, Soot particle size distributions in a well-stirred reactor/plug flow reactor, Proceedings of Combustion Institute 31 (2007) 675.

[8] B. Collignon, P. N. M. Hoang, S. Picaud, D. Liotard, M. T. Rayez, J. C. Rayez, A semi-empirical potential model for calculating interactions between large aromatic molecules and graphite surfaces, J. Mol. Struct.: THEOCHEM 772 (2006) 1-12.

[9] J. D. Kubicki, Molecular simulations of benzene and pah interactions with soot, Environ. Sci. Technol. 40(7) (2006) 2298-2303. 
[10] S. D. Chakarova-Käck, E. Schröder, B. I. Lundqvist, D. C. Langreth, Application of van der waals density functional to an extended systems: Adsorption of benzene and naphthalene on graphite, Phys. Rev. Letts. 96 (2006) 146107.

[11] P. A. Elkington, G. Curthoys, Heats of adsorption on carbon black surfaces, J. Phys. Chem. 73 (1969) 2321.

[12] A. J. Fisher, P. E. Blöchl, Adsorption and scanning-tunnelingmicroscope imaging of benzene on graphite and $\operatorname{mos}_{2}$, Phys. Rev. Lett. 70 (1993) 3263.

[13] S. D. Chakarova, E. Schröder, van der waals interactions of the benzene dimer: Towards treatment of polycyclic aromatic hydrocarbon dimers, Materials Science and Engineering: C 25(5-8) (2004) 787-792.

[14] C. Pierce, Localized adsorption on graphite and absolute surface areas, J. Phys. Chem. 73 (1969) 813.

[15] L. A. Girifalco, M. Hodak, R. S. Lee, Carbon nanotubes, buckyballs, ropes, and a universal graphitic potential, Phys. Rev. B 62 (2000) 13104.

[16] W. L. Jorgensen, D. L. Severance, Aromatic-aromatic interactions: Free energy profiles for the benzene dimer in water, chloroform, and liquid benzene, J. Am. Chem. Soc 112 (1990) 4768-4774.

[17] B. J. Cox, N. Thamwattana, J. M. Hill, Orientation of spheroidal fullerene inside carbon nanotubes with potential applications as memory devices in nano-computing, J. Phys. A : Math. Theor. 41 (2008) 235209 .

[18] C. A. Hunter, K. R. Lawson, J. Perkins, C. J. Urch, Aromatic interactions, J. Chem. Soc., Perkin Trans 2 (2001) 651-669.

[19] R. Zacharia, H. Ulbricht, T. Hertel, Interlayer cohesive energy of graphite from thermo desorption of polyaromatic hydrocarbons, Phys. Rev. B 69 (2004) 155406. 